\title{
Density-driven instabilities of miscible fluids in a capillary tube: linear stability analysis
}

\author{
By SURYA HARITH VANAPARTHY, E. MEIBURG \\ AND D. WILHELM† \\ Department of Mechanical and Environmental Engineering, University of California, \\ Santa Barbara, CA 93106, USA
}

(Received 24 February 2003 and in revised form 15 July 2003)

A linear stability analysis is presented for the miscible interface formed by placing a heavier fluid above a lighter one in a vertically oriented capillary tube. The analysis is based on the three-dimensional Stokes equations, coupled to a convectiondiffusion equation for the concentration field, in cylindrical coordinates. A generalized eigenvalue problem is formulated, whose numerical solution yields both the growth rate and the two-dimensional eigenmodes as functions of the governing parameters in the form of a Rayleigh number and a dimensionless interfacial thickness. The dispersion relations show that for all values of the governing parameters the threedimensional mode with an azimuthal wavenumber of 1 represents the most unstable disturbance. The stability results also indicate the existence of a critical Rayleigh number of about 920, below which all perturbations are stable. The growth rates are seen to reach a plateau for Rayleigh numbers in excess of $10^{6}$. In order to analyse the experimental observations by Kuang et al. (2002), which show that a small amount of net flow can stabilize the azimuthal instability mode and maintain an axisymmetric evolution, a base flow of Poiseuille type is included in the linear stability analysis. Results show that a weak base flow leads to a slight reduction of the growth rates of both axisymmetric and azimuthal modes. However, within the velocity interval that could be analysed in the present investigation, there is no indication that the axisymmetric mode overtakes its azimuthal counterpart.

\section{Introduction}

The capillary tube represents one of the fundamental configurations historically employed in investigations of interfacial phenomena and diffusive effects in the region of contact between two fluids. Both hydrodynamic stability problems and displacement processes have been studied extensively in this geometry. Hales (1937) was among the first to address the stability of an unstably stratified, variable-density fluid mixture with a constant density gradient in a vertically oriented capillary tube, in the absence of any net flow through the tube. He found that a stable equilibrium is possible, as long as the density gradient does not exceed a certain critical value. Taylor (1954) devised a simple experiment to obtain this critical gradient of density, which he argued could be used to determine the diffusion coefficient of a fluid pair, cf. also the recent related experimental work by Debacq et al. (2001). Wooding (1959) took 
an analytical approach to the stability problem for a constant density gradient in a capillary tube. He observed that the three-dimensional mode $\beta=1$, where $\beta$ denotes the azimuthal wavenumber, represents the most unstable disturbance. Batchelor \& Nitsche (1993) extended the above analyses to base states involving density profiles that vary sinusoidally in the vertical direction or deviate from a constant value only in a central layer of small vertical extent. They confirm that $\beta=1$ represents the most unstable mode for the uniform density gradient. Without proof, they assume that this also holds for the case of sinusoidally varying density. All of the above investigations were limited to cases in which viscosity variations are absent. To our knowledge, the stability of the situation that can be realized most easily in an experiment, namely that of a relatively thin, miscible interface formed by placing a heavier fluid above a lighter one in a capillary tube, has not yet been addressed from a theoretical point of view. This is the configuration to be analysed in the present investigation.

The presence of a net flow through the tube complicates the situation considerably. The investigations by Taylor (1960) and Cox (1962) discussed the fractional amount of viscous fluid left behind on the wall of a tube when it is expelled by an inviscid fluid with which it is immiscible, as a function of a suitably defined capillary number $C a$. Density effects were deemed unimportant in these studies. Reinelt \& Saffman (1985) presented numerical calculations for this case, which show very good agreement with the experimental observations. This classical work has recently been extended to finite viscosity ratios in the work of Soares, Carvalho \& Souza Mendes (2002). Petitjeans \& Maxworthy (1996) along with Chen \& Meiburg (1996) analysed the corresponding miscible problem both experimentally and computationally, based on the Stokes equations, cf. also the related experiments by Scoffoni, Lajeunesse \& Homsy (2000). In these flows, a cutoff length is set by diffusive effects rather than surface tension, so that in some sense a Péclet number $\mathrm{Pe}$ takes the place of $\mathrm{Ca}$. The above authors also address the role of density differences by conducting experiments and simulations in vertical tubes. Substantial differences between the experiments and the numerical data are observed at small values of $P e$, in that a quasi-steady finger emerges for significantly smaller values of $P e$ in the simulations, compared to the experiments. This raises the question as to whether non-conventional, so-called Korteweg stresses (Korteweg 1901; Joseph \& Renardy 1992) or divergence effects can be important, an issue that has been addressed by Chen \& Meiburg (2002). A particularly striking finding was reported in the follow-up experiments of Kuang, Maxworthy \& Petitjeans (2002). In a vertical capillary tube without net flow, these authors observe that the sharp interface formed by placing a heavier, more viscous silicone oil above a lighter and less viscous one leads to an interfacial instability with an azimuthal wavenumber $\beta=1$. However, when a small upward net flow was applied to this gravitationally unstable base state, the interface evolved in an axisymmetric fashion, rather than exhibiting an azimuthal instability mode. It is at present unclear whether this reflects an effect of the net flow within the linear framework of the stability problem, or if it represents a nonlinear effect. This is one of the questions to be addressed below. Furthermore, it is unclear to what extent the above observation depends on the viscosity ratio of the two fluids.

In the following, we investigate the stability of a thin miscible interface formed by placing a heavier fluid on top of a lighter one. This Rayleigh-Taylor instability has been studied previously in unbounded domains. For a review of that literature, see Batchelor \& Nitsche (1991). Here we will focus on the geometry of a vertical capillary tube. The analysis will be based on the three-dimensional Stokes equations, and it will proceed along similar lines to our recent investigation for the corresponding 


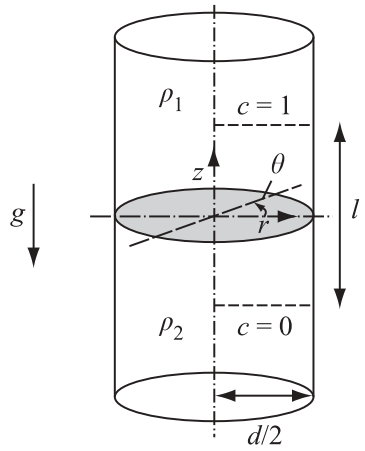

FiguRE 1. Sketch of the vertical capillary tube and the cylindrical coordinate system.

situation in a Hele-Shaw cell, cf. Graf, Meiburg \& Härtel (2002) as well as the related experiments and nonlinear simulations reported by Fernandez et al. (2002). In $\S 2$ the physical problem, along with the governing equations and their nondimensionalization, is presented. The linearization is described for both axisymmetric and azimuthal perturbations, and the numerical procedure for solving the resulting eigenvalue problem is outlined. In $\S 3$, the results of the stability analysis will be presented in the form of dispersion relations and associated information. Section 4 discusses the framework employed to investigate the linear stability of the interface in the presence of a weak axial net flow. The main conclusions will be summarized in $\S 5$.

\section{Physical problem}

\subsection{Governing equations}

We consider the situation of a heavier fluid placed above a lighter one in a vertically oriented capillary tube of diameter $d$, cf. figure 1 . The two fluids are miscible in all proportions. We assume a suitably defined Reynolds number to be small, so that their motion is governed by the three-dimensional Stokes equations

$$
\begin{gathered}
\nabla \cdot \boldsymbol{u}=0, \\
\nabla p=\mu \nabla^{2} \boldsymbol{u}+\rho \boldsymbol{g}, \\
\frac{\partial c}{\partial t}+\boldsymbol{u} \cdot \nabla \boldsymbol{c}=D \nabla^{2} c .
\end{gathered}
$$

These equations describe the conservation of mass, momentum and species, respectively. Here $\boldsymbol{u}$ represents the flow velocity, $\boldsymbol{g}$ denotes the vector of the gravitational acceleration, which is taken to point in the $-z$-direction, and $c$ indicates the concentration of the heavier fluid. The viscosity $\mu$ and the molecular diffusion coefficient $D$ are considered constant throughout the mixture. Note that implicitly contained in the above set of equations is the Boussinesq approximation, which assumes that density variations are significant in the gravitational term only. The density $\rho$ is taken to be a linear function of the concentration $c$ :

$$
\rho=\rho_{2}+c\left(\rho_{1}-\rho_{2}\right),
$$

which is valid for small density variations. Here $\rho_{1}$ and $\rho_{2}$ represent the density of the heavier and lighter fluids, respectively. To render the governing equations dimensionless, we introduce a characteristic length $L^{*}$, velocity $U^{*}$, time $T^{*}$, pressure 
$P^{*}$ and density difference $R^{*}$ defined as

$$
\begin{gathered}
L^{*}=d, \\
U^{*}=\frac{\Delta \rho g d^{2}}{\mu}, \\
T^{*}=\frac{\mu}{\Delta \rho g d}, \\
P^{*}=\Delta \rho g d, \\
R^{*}=\Delta \rho=\rho_{1}-\rho_{2} .
\end{gathered}
$$

By using these relations in equations (2.1) to (2.3), we obtain the dimensionless set of equations

$$
\begin{gathered}
\nabla \cdot \boldsymbol{u}=0, \\
\nabla p=\nabla^{2} \boldsymbol{u}-c \nabla z, \\
\frac{\partial c}{\partial t}+\boldsymbol{u} \cdot \nabla \boldsymbol{c}=\frac{1}{R a} \nabla^{2} c,
\end{gathered}
$$

where the Rayleigh number $R a$ is defined as

$$
R a=\frac{\Delta \rho g d^{3}}{D \mu}
$$

The Stokes equations, when formulated in cylindrical coordinates $(r, \theta, z)$, exhibit terms of the type $r^{-1}, r^{-2}, r^{-3}$, which lead to geometric singularities at the axis $r=0$. Verzicco \& Orlandi (1996) propose rewriting the governing equations by replacing the velocity components $v_{r}, v_{\theta}$ and $v_{z}$ with $q_{r}=v_{r} r, q_{\theta}=v_{\theta}$ and $q_{z}=v_{z}$, respectively. Thus $q_{r}=0$ is by definition on the axis, which on a staggered grid avoids the problem of the singularities. The authors also demonstrate that alternative formulations (e.g. $\left.q_{\theta}=v_{\theta} r\right)$ neither enhance the accuracy nor simplify the discretization. In terms of the new variables $q_{r}, q_{\theta}$ and $q_{z}$, the dimensionless continuity equation (2.10) becomes

$$
\frac{\partial q_{r}}{\partial r}+\frac{\partial q_{\theta}}{\partial \theta}+r \frac{\partial q_{z}}{\partial z}=0 .
$$

The non-dimensional momentum equations (2.11) follow as

$$
\begin{gathered}
r \frac{\partial p}{\partial r}=r \frac{\partial}{\partial r}\left(\frac{1}{r} \frac{\partial q_{r}}{\partial r}\right)+\frac{1}{r^{2}} \frac{\partial^{2} q_{r}}{\partial \theta^{2}}+\frac{\partial^{2} q_{r}}{\partial z^{2}}-\frac{2}{r} \frac{\partial q_{\theta}}{\partial \theta} \\
\frac{1}{r} \frac{\partial p}{\partial \theta}=\frac{\partial}{\partial r}\left(\frac{1}{r} \frac{\partial}{\partial r}\left(r q_{\theta}\right)\right)+\frac{1}{r^{2}} \frac{\partial^{2} q_{\theta}}{\partial \theta^{2}}+\frac{\partial^{2} q_{\theta}}{\partial z^{2}}+\frac{2}{r^{3}} \frac{\partial q_{r}}{\partial \theta} \\
\frac{\partial p}{\partial z}=\frac{1}{r} \frac{\partial}{\partial r}\left(r \frac{\partial q_{z}}{\partial r}\right)+\frac{1}{r^{2}} \frac{\partial^{2} q_{z}}{\partial \theta^{2}}+\frac{\partial^{2} q_{z}}{\partial z^{2}}-c
\end{gathered}
$$

and the species conservation equation (2.12) takes the form

$$
\frac{\partial c}{\partial t}+\frac{q_{r}}{r} \frac{\partial c}{\partial r}+\frac{q_{\theta}}{r} \frac{\partial c}{\partial \theta}+q_{z} \frac{\partial c}{\partial z}=\frac{1}{R a}\left(\frac{1}{r} \frac{\partial}{\partial r}\left(r \frac{\partial c}{\partial r}\right)+\frac{1}{r^{2}} \frac{\partial^{2} c}{\partial \theta^{2}}+\frac{\partial^{2} c}{\partial z^{2}}\right) .
$$

The appropriate boundary conditions will be discussed below. 
2.2. Linearization and formulation of the eigenvalue problem

We linearize the above set of equations around a quiescent base state

$$
\left(\begin{array}{c}
q_{r} \\
q_{\theta} \\
q_{z} \\
p \\
c
\end{array}\right)(r, \theta, z, t)=\left(\begin{array}{c}
0 \\
0 \\
0 \\
\bar{p} \\
\bar{c}
\end{array}\right)(z)+\left(\begin{array}{l}
q_{r}^{\prime} \\
q_{\theta}^{\prime} \\
q_{z}^{\prime} \\
p^{\prime} \\
c^{\prime}
\end{array}\right)(r, \theta, z, t)
$$

where the base concentration profile is given by

$$
\bar{c}=0.5+0.5 \operatorname{erf}\left(\frac{z}{\delta}\right) .
$$

The parameter $\delta$ denotes the thickness of the interfacial region. We assume that the diffusive time scale of the base state is much larger than the characteristic time scale of the instability growth, so that the base state can be held constant for the purpose of evaluating the instability growth rate. For the purpose of quantifying this assumption, we define an instantaneous characteristic time scale of the self-similar diffusive growth of the base state in the form of $\delta^{2} / D$. A time scale for the growth of the perturbation is given as $\sigma^{-1}$, where $\sigma$ is the growth rate evaluated from the linear stability analysis. Consequently, the assumption of a quasi-steady base state is valid provided that the dimensional quantities satisfy

$$
\sigma \gg \frac{D}{\delta^{2}}
$$

In dimensionless form, this criterion becomes

$$
\sigma \delta^{2} \gg \frac{1}{R a} \text {. }
$$

The perturbations, denoted by a prime, are assumed to be of the form

$$
\left(\begin{array}{l}
q_{r}^{\prime} \\
q_{\theta}^{\prime} \\
q_{z}^{\prime} \\
p^{\prime} \\
c^{\prime}
\end{array}\right)(r, \theta, z, t)=\left(\begin{array}{c}
\hat{q}_{r}(r, z) \cos (\beta \theta) \\
\hat{q}_{\theta}(r, z) \sin (\beta \theta) \\
\hat{q}_{z}(r, z) \cos (\beta \theta) \\
\hat{p}(r, z) \cos (\beta \theta) \\
\hat{c}(r, z) \cos (\beta \theta)
\end{array}\right) \mathrm{e}^{\sigma t}
$$

where the hatted quantities represent the axisymmetric eigenfunctions, and $\beta$ denotes the azimuthal wavenumber. It should be noted that, due to the underlying geometry of the problem, only integral values of $\beta$ have physical significance. By substituting the above relations into the dimensionless conservation equations, subtracting out the base state and linearizing, we obtain the system of linear equations

$$
\begin{gathered}
\frac{\partial \hat{q}_{r}}{\partial r}+\beta \hat{q}_{\theta}+r \frac{\partial \hat{q}_{z}}{\partial z}=0 \\
r \frac{\partial \hat{p}}{\partial r}=r \frac{\partial}{\partial r}\left(\frac{1}{r} \frac{\partial \hat{q}_{r}}{\partial r}\right)-\frac{\beta^{2}}{r^{2}} \hat{q}_{r}+\frac{\partial^{2} \hat{q}_{r}}{\partial z^{2}}-\frac{2 \beta}{r} \hat{q}_{\theta}, \\
-\frac{\beta}{r} \hat{p}=\frac{\partial}{\partial r}\left(\frac{1}{r} \frac{\partial}{\partial r}\left(r \hat{q}_{\theta}\right)\right)-\frac{\beta^{2}}{r^{2}} \hat{q}_{\theta}+\frac{\partial^{2} \hat{q}_{\theta}}{\partial z^{2}}-\frac{2 \beta}{r^{3}} \hat{q}_{r},
\end{gathered}
$$




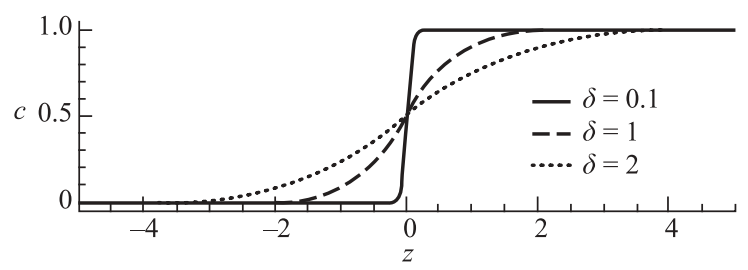

FIGURE 2. Base concentration profile $\bar{c}(z)$ for different values of the interfacial thickness $\delta$.

\begin{tabular}{ccc}
\hline$\beta$ & $l$ & $\sigma_{\max }$ \\
1 & 6 & $2.40421 \times 10^{-2}$ \\
& 8 & $2.40384 \times 10^{-2}$ \\
& 10 & $2.41510 \times 10^{-2}$ \\
2 & 6 & $1.75770 \times 10^{-2}$ \\
& 8 & $1.74593 \times 10^{-2}$ \\
& 10 & $1.75911 \times 10^{-2}$
\end{tabular}

TABLE 1. The maximum eigenvalue $\sigma_{\max }$ as a function of $l$, for different $\beta$, with $\delta=0.1$ and $R a=10^{7}$.

$$
\begin{gathered}
\frac{\partial \hat{p}}{\partial z}=\frac{1}{r} \frac{\partial}{\partial r}\left(r \frac{\partial \hat{q}_{z}}{\partial r}\right)-\frac{\beta^{2}}{r^{2}} \hat{q}_{z}+\frac{\partial^{2} \hat{q}_{z}}{\partial z^{2}}-\hat{c}, \\
\sigma \hat{c}+\hat{q}_{z} \frac{\partial \bar{c}}{\partial z}=\frac{1}{R a}\left(\frac{1}{r} \frac{\partial}{\partial r}\left(r \frac{\partial \hat{c}}{\partial r}\right)-\frac{\beta^{2}}{r^{2}} \hat{c}+\frac{\partial^{2} \hat{c}}{\partial z^{2}}\right),
\end{gathered}
$$

which represent an eigenvalue problem with $\hat{p}, \hat{q}_{r}, \hat{q}_{\theta}, \hat{q}_{z}$ and $\hat{c}$ as eigenfunctions and $\sigma$ as the eigenvalue of the system. There are two externally prescribed parameters in the form of the Rayleigh number $R a$ and the interfacial thickness $\delta$.

\subsection{Numerical implementation of three-dimensional perturbations}

The computational domain for the solution of the eigenvalue problem extends from the axis to the outer wall in the $r$-direction, i.e. from 0 to 0.5 , and from $-l / 2$ to $l / 2$ in the vertical $z$-direction, as shown in figure 1 . The domain length $l$ has to be chosen sufficiently large for its effect on the numerical results for the eigenvalue and eigenfunctions to be negligible. Test calculations were carried out in order to establish the independence of the maximum eigenvalue $\sigma_{\max }$ from $l$, for different values of $\beta$ and different base concentration profiles, cf. figure 2 . It is found that beyond a certain value, $l$ no longer affects the value of $\sigma_{\max }$ in a significant way, as demonstrated for a typical parameter combination in table 1. The linear equations (2.24)-(2.28) are discretized by second-order finite differences in both the $r$ - and $z$-directions. In order to be able to concentrate the numerical resolution in the interfacial region, a non-equidistant grid is employed based on the mapping function given in Fletcher (1991):

$$
s=P \eta+(1-P)\left(1-\frac{\tanh [Q(1-\eta)]}{\tanh Q}\right) .
$$

We employ two separate subdomains for $z=s$ and $z=-s$, respectively. Depending on the specific value of $\delta$, we select the parameters $P$ and $Q$ such that an appropriate concentration of grid points is obtained in the interfacial region. The 
required numerical resolution is established by means of test calculations. These show that for most cases $N_{r}=19$ points in the radial direction are sufficient to keep the error in the eigenvalue below $0.1 \%$. The required number of points in the $z$ direction depends on the domain length and the interface thickness $\delta$. The largest calculations employ up to $N_{z}=91$ and $N_{r}=23$ points, which results in a matrix of size $5 N_{z} N_{r} \times 5 N_{z} N_{r}=10465 \times 10465$.

At the outer wall of the tube, i.e. at $r=0.5$, all velocity components are assumed to vanish, along with the normal derivative of the concentration perturbation. The vertical domain boundaries are sufficiently far away from the interface that we can prescribe homogeneous Dirichlet conditions for all velocity components, as well as for the concentration perturbation. At the axis, i.e. at $r=0, q_{r}=0$ because $q_{r}=v_{r} r$. We do not need to specify boundary conditions for the other velocity components as well as the concentration perturbation, at the axis, since the staggered grid implies only grid points corresponding to radial velocity lie on the axis. For the pressure variable, no boundary conditions are necessary, as we employ a staggered grid.

Upon discretization, the system of linear equations (2.24)-(2.28) can be written in matrix form as

$$
\boldsymbol{A} \boldsymbol{\phi}=\sigma \boldsymbol{B} \boldsymbol{\phi}
$$

where the matrices $\boldsymbol{A}, \boldsymbol{B}$ and the eigenvector $\boldsymbol{\phi}$ are of the following form:

$$
\left(\begin{array}{ccccc}
0 & \frac{\partial}{\partial r} & \beta & r \frac{\partial}{\partial z} & 0 \\
-r \frac{\partial}{\partial r} & \mathbf{M}_{1} & -\frac{2 \beta}{r} & 0 & 0 \\
\frac{\beta}{r} & -\frac{2 \beta}{r^{2}} & \mathbf{M}_{2} & 0 & 0 \\
-\frac{\partial}{\partial z} & 0 & 0 & \mathbf{M}_{3} & -\mathbf{I} \\
0 & 0 & 0 & -\frac{\partial \bar{c}}{\partial z} & \mathbf{M}_{4}
\end{array}\right)\left(\begin{array}{c}
\hat{p} \\
\hat{q}_{r} \\
\hat{q}_{\theta} \\
\hat{q}_{z} \\
\hat{c}
\end{array}\right)=\sigma\left(\begin{array}{lllll}
0 & 0 & 0 & 0 & 0 \\
0 & 0 & 0 & 0 & 0 \\
0 & 0 & 0 & 0 & 0 \\
0 & 0 & 0 & 0 & 0 \\
0 & 0 & 0 & 0 & \mathbf{I}
\end{array}\right)\left(\begin{array}{c}
\hat{p} \\
\hat{q}_{r} \\
\hat{q}_{\theta} \\
\hat{q}_{z} \\
\hat{c}
\end{array}\right) .
$$

Here

$$
\begin{gathered}
\mathbf{M}_{1}=r \frac{\partial}{\partial r}\left(\frac{1}{r} \frac{\partial}{\partial r}\right)-\frac{\beta^{2}}{r^{2}}+\frac{\partial^{2}}{\partial z^{2}}, \\
\mathbf{M}_{2}=\frac{\partial}{\partial r}\left(\frac{1}{r} \frac{\partial}{\partial r} r\right)-\frac{\beta^{2}}{r^{2}}+\frac{\partial^{2}}{\partial z^{2}} \\
\mathbf{M}_{3}=\frac{1}{r} \frac{\partial}{\partial r}\left(r \frac{\partial}{\partial r}\right)-\frac{\beta^{2}}{r^{2}}+\frac{\partial^{2}}{\partial z^{2}} \\
\mathbf{M}_{4}=\frac{1}{R a}\left(\frac{1}{r} \frac{\partial}{\partial r}\left(r \frac{\partial}{\partial r}\right)-\frac{\beta^{2}}{r^{2}}+\frac{\partial^{2}}{\partial z^{2}}\right) .
\end{gathered}
$$

This system has to be solved numerically in order to determine the eigenvalue $\sigma$ along with the corresponding eigenfunctions $\hat{q}_{r}, \hat{q}_{\theta}, \hat{q}_{z}, \hat{p}$ and $\hat{c}$, as functions of the azimuthal wavenumber $\beta$, for the two parameters characterizing the overall fluid system and the base state, $R a$ and $\delta$.

To keep the computational effort in an acceptable range the numerical eigenvalue problem is solved iteratively for the leading eigenvalues by an Arnoldi method (Sorensen 1992). For the computational implementation we make use of the public 


\begin{tabular}{lcc}
\multicolumn{1}{c}{ Re } & $\sigma_{\max }$ & $\xi$ \\
0 & $7.79497 \times 10^{-3}$ & $3.38486 \times 10^{+07}$ \\
0.001 & $7.79497 \times 10^{-3}$ & $6.64375 \times 10^{-11}$ \\
0.01 & $7.79496 \times 10^{-3}$ & $6.64588 \times 10^{-11}$ \\
0.1 & $7.79486 \times 10^{-3}$ & $7.20705 \times 10^{-11}$
\end{tabular}

TABLE 2. Eigenvalues and eigenfunction residuals for $\delta=1, R a=10^{7}$ and $\beta=1$ and various values of $R e$. The results indicate that a value of $R e=0.001$ has a negligible effect on the eigenvalue, but leads to much better convergence of the eigenfunctions.

domain software package ARPACK (Maschhoff \& Sorensen 1996). Test calculations and comparisons with direct solvers for a system of linear equations (LAPACK) indicate that in this way the eigenvalues can be computed to a high degree of accuracy. However, at high $R a$ the corresponding eigenfunctions are not always fully converged and the overall rate of convergence is quite slow at times. Consequently, we follow the suggestion by Graf et al. (2002), and to accelerate the convergence and improve the accuracy modify the original system of equations slightly to

$$
\begin{gathered}
\nabla \cdot \boldsymbol{u}=0, \\
R e \frac{\partial \boldsymbol{u}}{\partial t}+\nabla p=\nabla^{2} \boldsymbol{u}-c \nabla z, \\
\frac{\partial c}{\partial t}+\boldsymbol{u} \cdot \nabla \boldsymbol{c}=\frac{1}{R a} \nabla^{2} c,
\end{gathered}
$$

where Re represents a Reynolds-number-like parameter. Test results shown in table 2 for $\delta=1, \beta=1, R a=10^{7}$ and various values of $R e$, demonstrate that for $R e=0.001$ the magnitude of the eigenvalue is essentially unaffected by $R e$, whereas the eigenfunctions are much better converged than for $R e=0$, as indicated by the value of the residual $\xi$. Consequently, this value of $R e$ is used in all the calculations.

\subsection{Numerical implementation of axisymmetric perturbations}

In order to obtain information on the stability of purely axisymmetric perturbations, we consider the case of $\beta=0$ separately. To avoid boundary conditions for pressure and also to save memory by reducing the total number of variables used in the computation, we can conveniently rewrite the governing equations in the streamfunction and vorticity variables:

$$
\begin{gathered}
\nabla^{2} \psi+\omega=0, \\
R e \frac{\partial \omega}{\partial t}=\nabla^{2} w+\frac{\partial c}{\partial r}, \\
\frac{\partial c}{\partial t}+\boldsymbol{u} \cdot \nabla \boldsymbol{c}=\frac{1}{R a} \nabla^{2} c .
\end{gathered}
$$

Vorticity $\omega$ and streamfunction $\psi$ are defined, in the usual way, as

$$
\begin{gathered}
v_{r}=-\frac{1}{r} \frac{\partial \psi}{\partial z}, \quad v_{z}=\frac{1}{r} \frac{\partial \psi}{\partial r}, \\
\omega=\frac{\partial v_{r}}{\partial z}-\frac{\partial v_{z}}{\partial r} .
\end{gathered}
$$




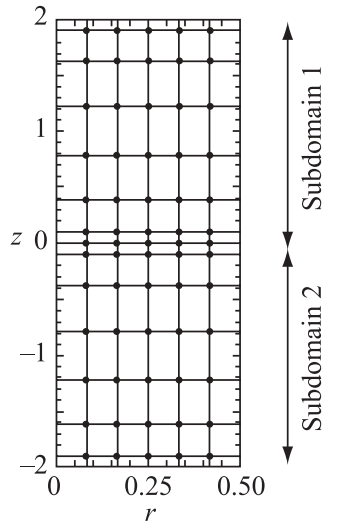

FIGURE 3. Example of mesh point distribution for the axisymmetric problem, with a Chebyshev grid in the $z$-direction and compact finite differences in the $r$-direction.

We assume an axisymmetric disturbance of the form

$$
\left(\begin{array}{c}
\psi^{\prime} \\
\omega^{\prime} \\
c^{\prime}
\end{array}\right)(r, z, t)=\left(\begin{array}{l}
\hat{\psi}(r, z) \\
\hat{\omega}(r, z) \\
\hat{c}(r, z)
\end{array}\right) \mathrm{e}^{\sigma t},
$$

where $\hat{\psi}$ is set to zero on all domain boundaries; $\hat{\omega}$ vanishes on all boundaries except for the outer wall, where it takes the value $\left(\partial^{2} \hat{\psi} / \partial r^{2}\right) / r$. At the far-field boundaries the concentration perturbation is assumed to vanish, whereas along the outer wall and the tube axis its normal derivative $\partial \hat{c} / \partial r$ goes to zero. A staggered grid is not required here, due to the absence of the pressure variable, and of singularities at the axis. Hence, a Chebyshev collocation method is used in the z-direction with two separate sub-domains that cover the regions $z \geqslant 0$ and $z \leqslant 0$, respectively, in order to concentrate grid points at the interface. In the radial direction a highly accurate compact finite difference scheme of third order at the wall and up to tenth order in the interior is used, cf. Lele (1992). An example of the mesh point distribution is shown in figure 3.

By linearizing the system of equations (2.38)-(2.40) using (2.43), discretization leads to an eigenvalue system of the form

$$
\left(\begin{array}{ccc}
\mathbf{M}_{1} & \mathbf{I} & 0 \\
0 & \mathbf{M}_{2} & \frac{\partial}{\partial r} \\
-\frac{1}{r} \frac{\partial \bar{c}}{\partial z} \frac{\partial}{\partial r} & 0 & \mathbf{M}_{3}
\end{array}\right)\left(\begin{array}{l}
\hat{\psi} \\
\hat{\omega} \\
\hat{c}
\end{array}\right)=\sigma\left(\begin{array}{ccc}
0 & 0 & 0 \\
0 & \operatorname{Re} \mathbf{I} & 0 \\
0 & 0 & \mathbf{I}
\end{array}\right)\left(\begin{array}{c}
\hat{\psi} \\
\hat{\omega} \\
\hat{c}
\end{array}\right)
$$

with

$$
\begin{gathered}
\mathbf{M}_{1}=\frac{1}{r} \frac{\partial^{2}}{\partial r^{2}}-\frac{1}{r^{2}} \frac{\partial}{\partial r}+\frac{1}{r} \frac{\partial^{2}}{\partial z^{2}}, \\
\mathbf{M}_{2}=\frac{\partial}{\partial r}\left(\frac{1}{r} \frac{\partial}{\partial r} r\right)+\frac{\partial^{2}}{\partial z^{2}}, \\
\mathbf{M}_{3}=\frac{1}{R a}\left(\frac{1}{r} \frac{\partial}{\partial r}\left(r \frac{\partial}{\partial r}\right)+\frac{\partial^{2}}{\partial z^{2}}\right) .
\end{gathered}
$$




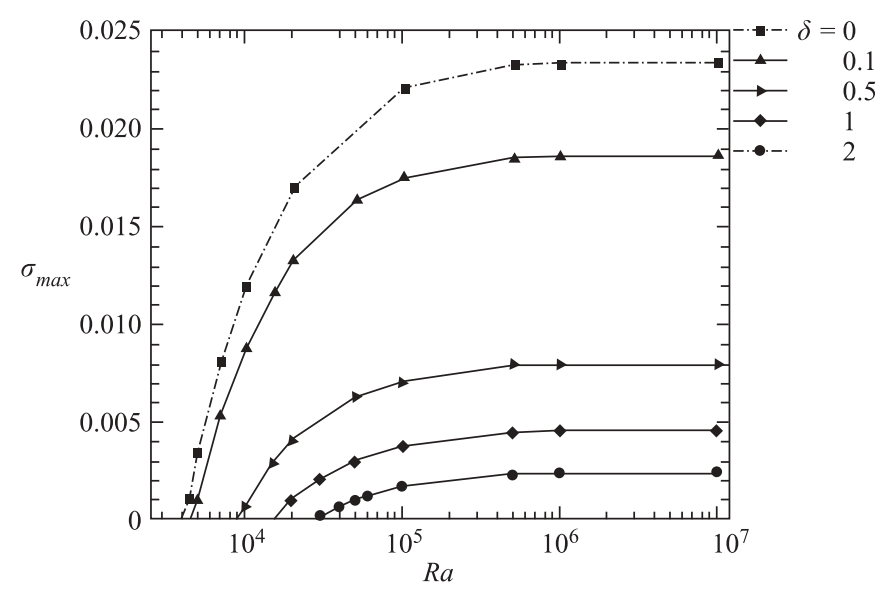

FIGURE 4. Axisymmetric perturbations: eigenvalue $\sigma$ as a function of $R a$, for different interface thicknesses $\delta$. The dashed line corresponds to extrapolated data for the step function profile.

\subsection{Validation}

The required numerical resolution along with the necessary vertical extent of the computational domain is established by means of convergence studies for test cases. In addition, the computational approach is validated by comparing the growth rates with those obtained from fully nonlinear, three-dimensional spectral simulations of variable-density miscible displacements in a capillary tube, cf. Wilhelm \& Meiburg (2003). For $\beta=0$ and 1 , respectively, those simulations yielded $\sigma \approx 0.013$ and 0.0195 , for $R a=1.35 \times 10^{5}$ and $\delta=0.2$. Our present data agree with those values to within less than $1 \%$. Finally, the growth rates obtained from the three-dimensional stability calculations in the limit $\beta \rightarrow 0$ are seen to agree closely with the corresponding values obtained from the axisymmetric stability calculations. This implies that the secondorder finite difference scheme for the three-dimensional problem is adequate for the numerical analysis of the problem at hand.

\section{Results}

\subsection{Axisymmetric perturbations}

We begin by discussing linear stability results for axisymmetric perturbations. The leading eigenvalue $\sigma$ is shown as a function of $R a$ for several values of the interface thickness parameter $\delta$ in figure 4. In general, thinner interfaces and larger $R a$ values are seen to be destabilizing. For $R a>10^{6}$, the growth rate is seen to asymptotically reach a plateau, whose value depends on $\delta$.

For each $\delta$, a critical value $R a_{\text {crit }}$ can be identified, below which the base state is stable to axisymmetric perturbations. The existence of this critical value reflects the stabilizing influence of the outer wall. The $R a_{\text {crit }}$ values found here for capillary tubes are significantly higher than those reported by Graf et al. (2002) for two-dimensional perturbations in Hele-Shaw cells. This is to be expected, as the stabilizing influence by the walls should be stronger in a capillary tube, where the axisymmetric perturbation is surrounded by the solid wall on all sides. In a Hele-Shaw cell, on the other hand, two-dimensional perturbations are affected by the walls only in the direction normal to the gap, but not in the spanwise direction. Figure 5 depicts the critical Rayleigh number $R a_{\text {crit }}$ as a function of $\delta$. For $0.1 \leqslant \delta \leqslant 2, R a_{\text {crit }}$ is seen to grow approximately 


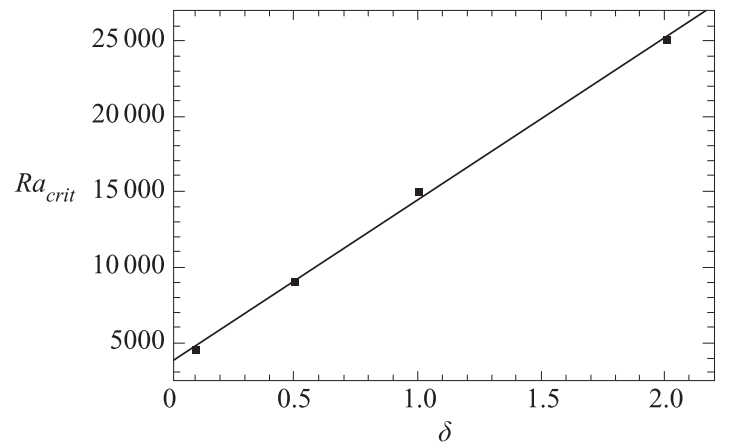

FIGURE 5. Axisymmetric perturbations: the critical Rayleigh number $R a_{\text {crit }}$ as a function of the interfacial thickness parameter $\delta$. The squares represent results of the stability calculations, while the straight line is a linear regression for these data. The results indicate that below $R a \approx 3700$, axisymmetric perturbations are stable even for a base state with a discontinuous jump in the concentration profile.

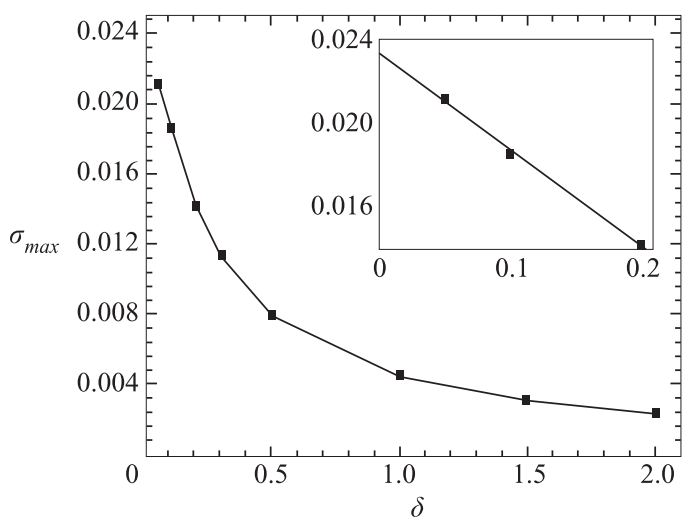

Figure 6. Axisymmetric perturbations: eigenvalue $\sigma$ as a function of $\delta$, for $R a=10^{7}$. The results indicate that the maximum growth rate for axisymmetric perturbations, achieved for a base state with a discontinuous jump in the concentration profile, is approximately 0.0234 .

linearly with the interface thickness. By linear regression, we obtain the relationship

$$
R a_{\text {crit }}=11000 \delta+3700 .
$$

This indicates that Rayleigh numbers below $O(3700)$ are always stable with respect to axisymmetric perturbations. By extrapolating the maximum growth rate, as a function of $\delta$ for $R a=10^{7}$, to $\delta=0$ we find that the maximum growth rate for axisymmetric perturbations in the presence of a step-like concentration base state is approximately 0.0234 , as shown in figure 6 . Figure 7 displays the concentration eigenfunctions associated with the largest eigenvalues, for $\delta=0.1$ and the two $R a$ values of $5 \times 10^{3}$ and $10^{7}$. One can clearly see a qualitative difference between the low- $R a$ results, for which the concentration perturbations reach a maximum near or at the walls, and the high- $R a$ counterpart which is characterized by nearly vanishing perturbation levels at the wall. This finding is similar to the observations of Graf et al. (2002) for the corresponding situation in a Hele-Shaw cell. Since the streamfunction and vorticity eigenfunctions for the two $R a$ values are very similar to each other (figure 8), the differences in the concentration eigenfunctions are predominantly due 

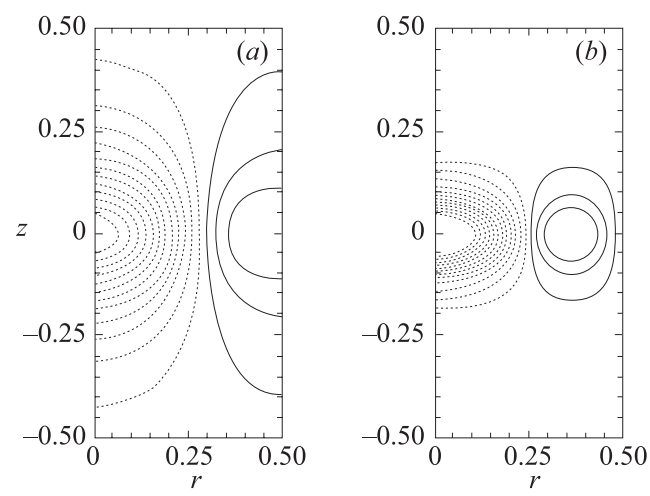

FIGURE 7. Axisymmetric perturbations: isocontours of the concentration eigenfunction $\hat{c}$ for $\delta=0.1$ and two different values of $R a:(a) 5 \times 10^{3},(b) 10^{7}$.

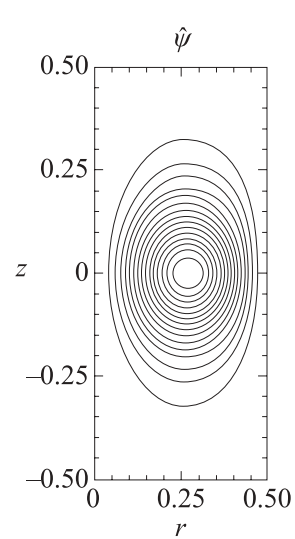

(a)

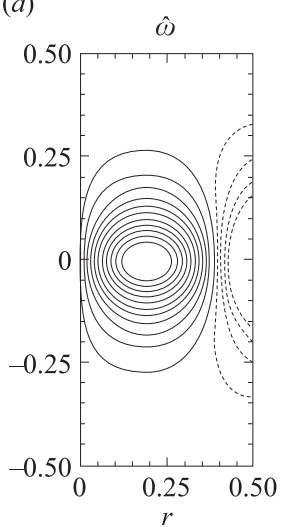

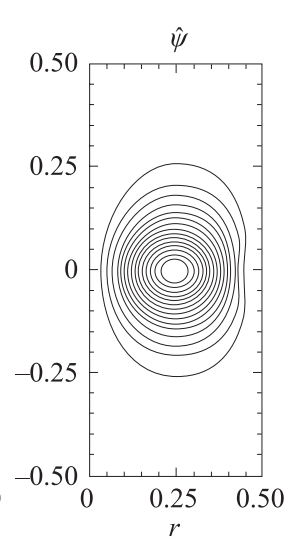

(b)

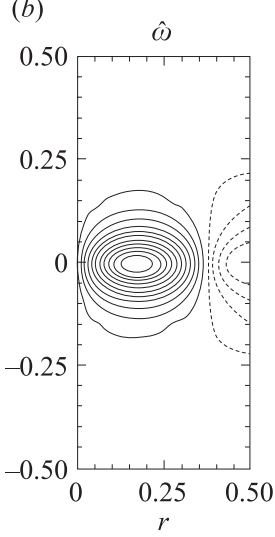

FIGURE 8 . Axisymmetric perturbations: streamfunction $\hat{\psi}$ and vorticity $\hat{\omega}$ eigenfunctions associated with the largest eigenvalue for $(a) R a=5 \times 10^{3}$ and $(b) R a=10^{7}$. The interfacial thickness parameter $\delta=0.1$.

to the effects of the diffusive term in the concentration equation. Qualitative sketches of nonlinear interfacial shapes corresponding to the high- and low- $R a$ concentration eigenfunctions are presented in figure 9. For low $R a$ values, the outer fluid layer is seen to propagate up or down the outer wall of the capillary tube, with a central finger propagating in the opposite direction along the axis. For higher Rayleigh numbers, we find the outer fluid layer to have its maximum propagation velocity away from the wall. Detailed parameter studies indicate that the eigenfunctions associated with the leading eigenvalue never give rise to more than one upward and one downward propagating fluid layer.

\subsection{Three-dimensional perturbations}

In figure 10 the leading eigenvalue is plotted as a function of the wavenumber $\beta$ for several $R a$ values, ranging from 500 to $10^{7}$, and a constant thickness of the interface $\delta=0.1$. These dispersion relationships show that for small and intermediate wavenumbers, the curves for $R a>10^{6}$ become indistinguishable, implying that a further increase in $R a$ affects only the range of unstable wavenumbers and the shortwavelength cutoff, but not the most dangerous wavenumber or its growth rate. It 

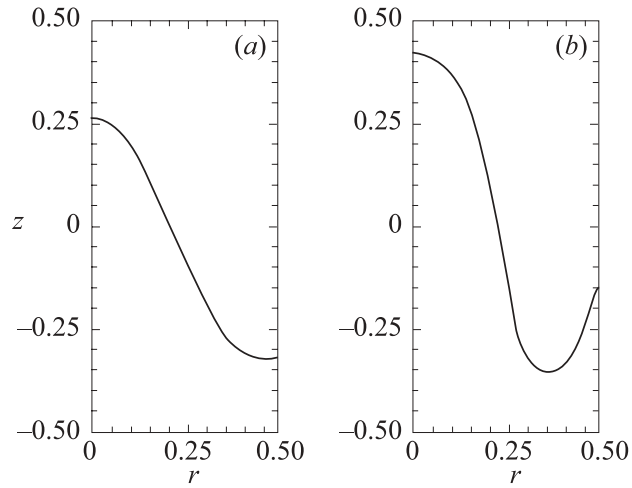

FIGURE 9. Axisymmetric perturbations: qualitative form of finite-amplitude fingers for $\delta=0.1$ and $(a) R a=5 \times 10^{3}$ and $(b) R a=10^{7}$.

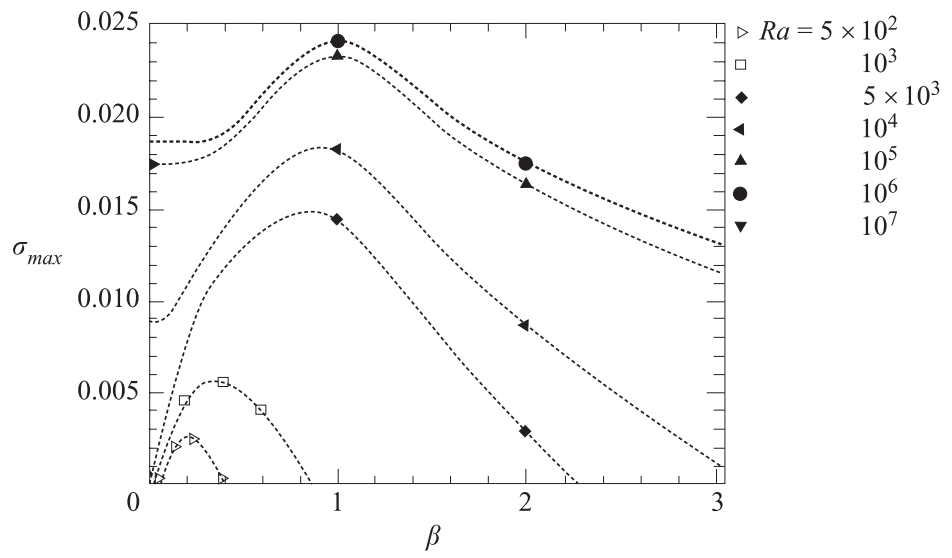

FIGURE 10. Three-dimensional perturbations: dispersion relationships for $\delta=0.1$ and various $R a$. For comparison, the axisymmetric data are plotted as well. The growth rate for $\beta=1$ is seen to exceed that for $\beta=0$ for all values of $R a$.

should be noted that the data for non-integer values of $\beta$ are plotted in figure 10 only in order to guide the eye, as only the integer values are of physical significance. Figure 10 demonstrates that the azimuthal perturbation $\beta=1$ is always more unstable than its axisymmetric counterpart. The concentration eigenfunctions for $\beta=0$ and 1 are shown in figure 11 for $\delta=0.1$ and $R a=10^{5}$. The presence of only one sign in the eigenfunction for $\beta=1$ indicates that the lighter fluid is rising in one half of the capillary tube, with the heavier fluid sinking in the other half.

The general shape of the eigenfunctions for the dominant azimuthal perturbations can be seen in figure 12. The three-dimensional nature of these perturbations is clear not only from the existence of an azimuthal velocity component $\hat{v}_{\theta}$, but also from the fact that for a given $\beta$ both the concentration $\hat{c}$ and the vertical velocity perturbation $\hat{v}_{z}$ are of one sign only. The qualitative form of the fingers produced, shown in figure 13, is similar to that inferred by Kuang et al. (2002), implying that there are two fingers in the capillary tube, one ascending and the other descending. Figure 14 shows that with increasing wavenumber, the shape of the eigenfunction remains similar, except that its maximum moves towards the outer wall. For 

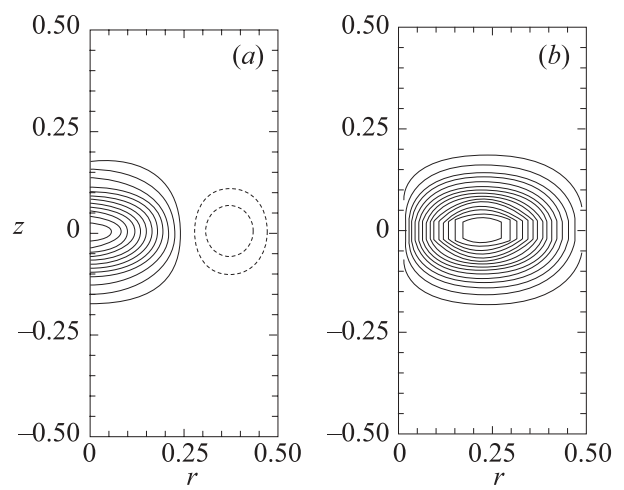

FIGURE 11. Isocontours of the concentration eigenfunction $\hat{c}$ for $(a) \beta=0$ and $(b) 1$, for $\delta=0.1$ and $R a=10^{5}$.
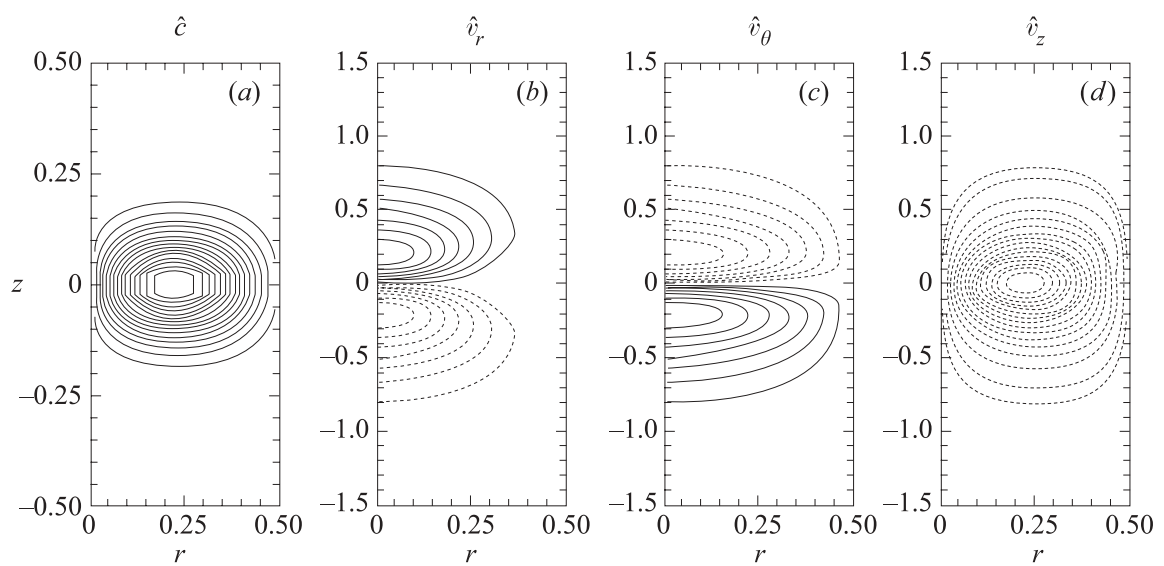

FIGURE 12. Concentration and velocity eigenfunction contours for the most dangerous wavenumber $\beta=1$, at $R a=10^{5}$ and $\delta=0.1$.

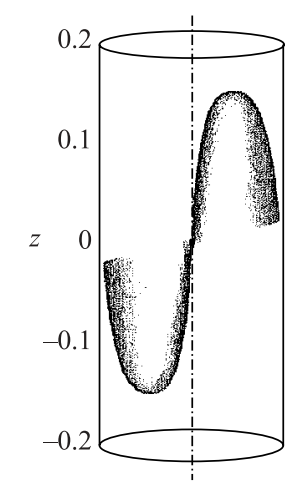

FIGURE 13. Three-dimensional perturbations: qualitative form of the finite-amplitude finger for $\beta=1, \delta=0.1$ and $R a=10^{7}$.

larger values of $\delta$, the corresponding dispersion relationships are presented in figure 15. We observe a general trend of decreasing growth rates and smaller cutoff wavenumbers for increasing interface thickness. However, there is no qualitative 

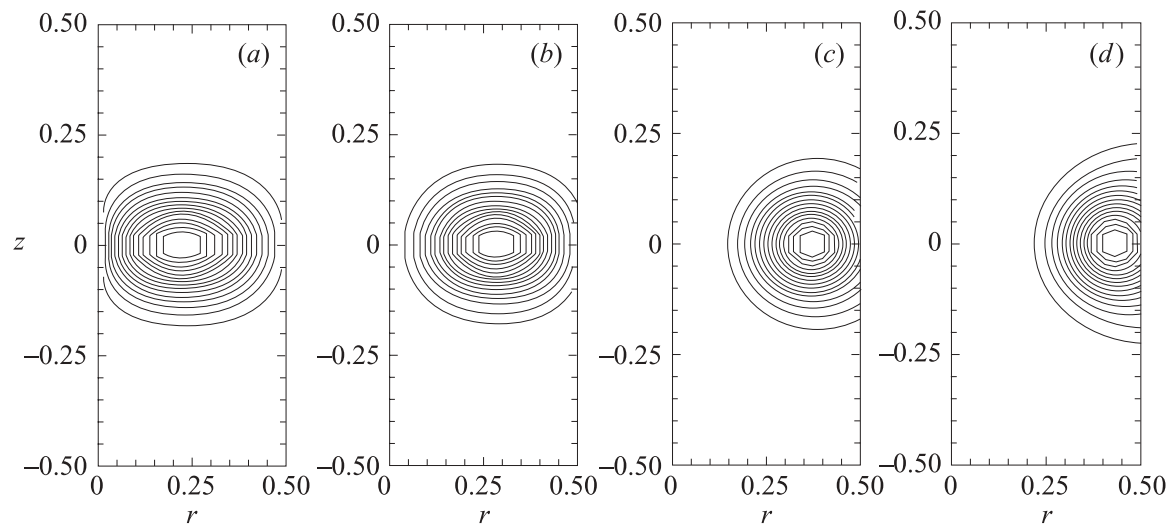

FIGURE 14. Isocontours of the concentration eigenfunction $\hat{c}$, for different wavenumbers $\beta$ : (a) $1,(b) 2,(c) 5,(d) 8$, at $R a=10^{5}$ and $\delta=0.1$. With increasing $\beta$, the maximum of the isocontours moves towards the outer wall.
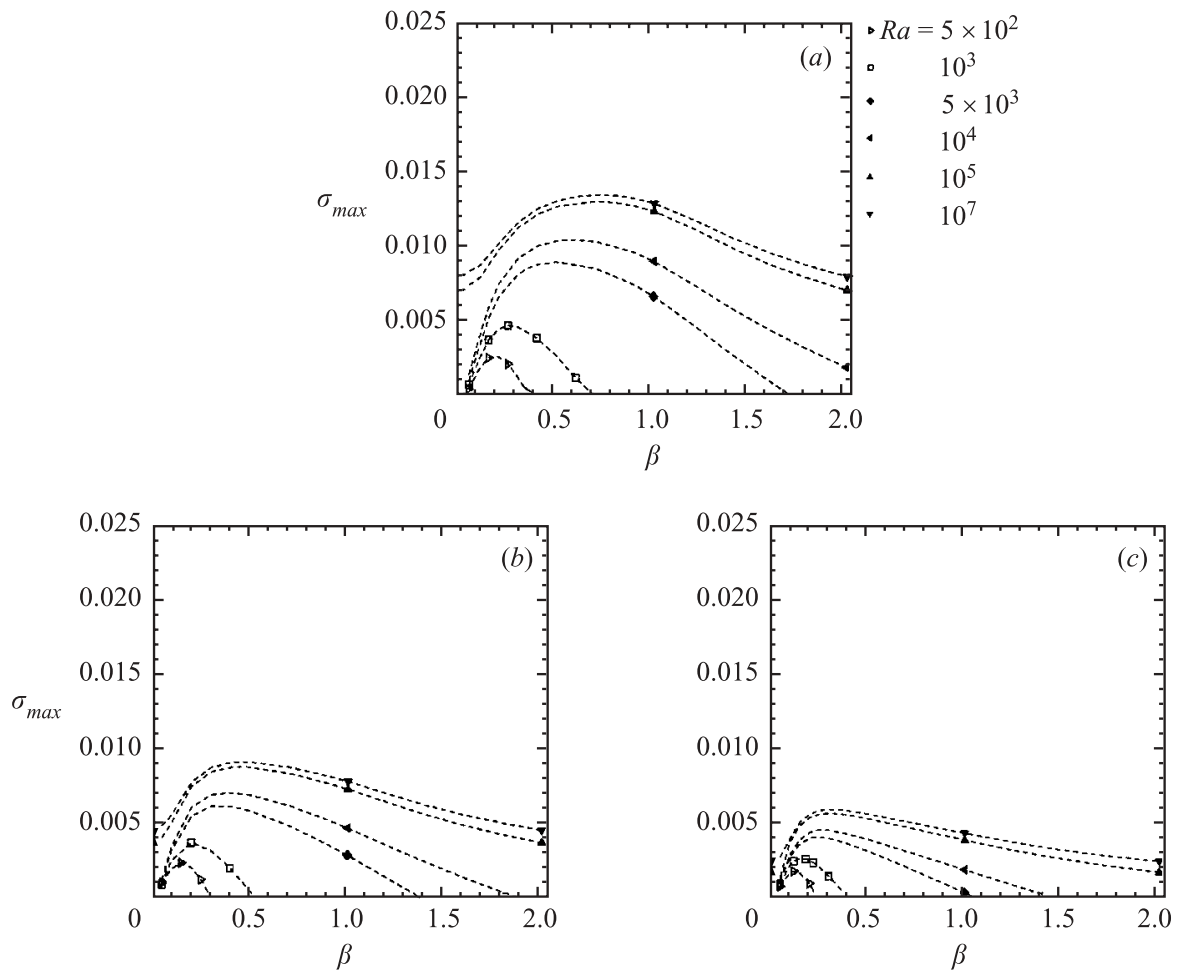

FigURE 15. Dispersion relationships for various interface thickness values: $(a) \delta=0.5,(b) 1$, (c) 2; and a range of Rayleigh numbers. $\beta=1$ represents the most amplified integer mode for all values of $\delta$ and $R a$.

change in the shape of the concentration eigenfunction $\hat{c}$ with increasing thickness, as shown in figure 16.

From the dispersion relationships shown in figures 10 and 15 we can deduce that for all values of the interface thickness, $\beta=1$ remains the most dangerous mode. For this reason, we will consider this wavenumber in more detail. Figure 17 depicts the 

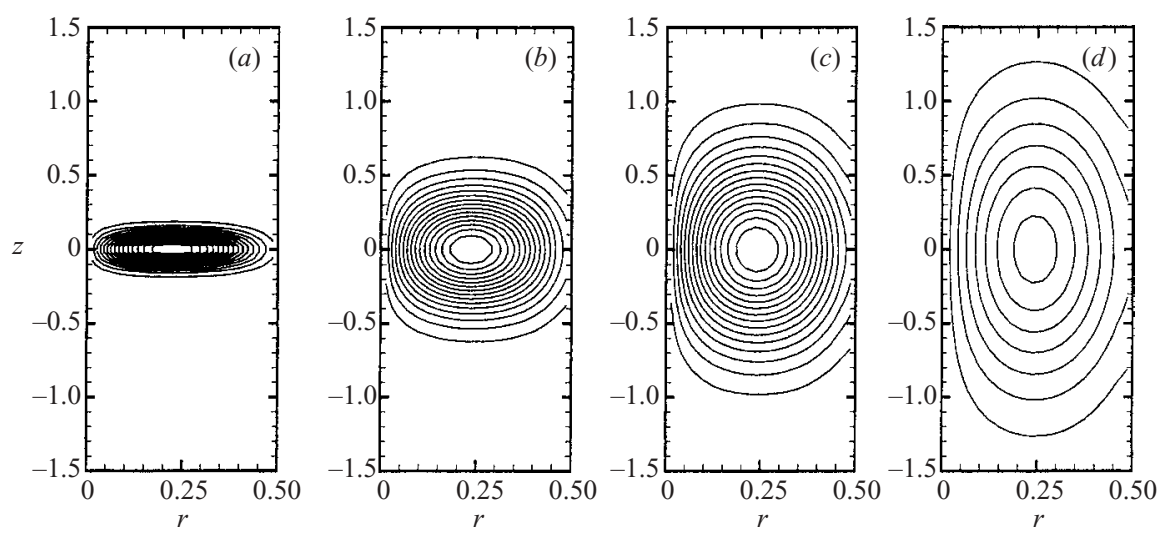

FIGURE 16. Isocontours of the concentration eigenfunction $\hat{c}$ for different values of $\delta:(a) 0.1$, (b) $0.5,(c) 1,(d) 2$, at $R a=10^{5}$ and $\beta=1$. While the isocontours become flatter for decreasing $\delta$, their shapes remain qualitatively the same.

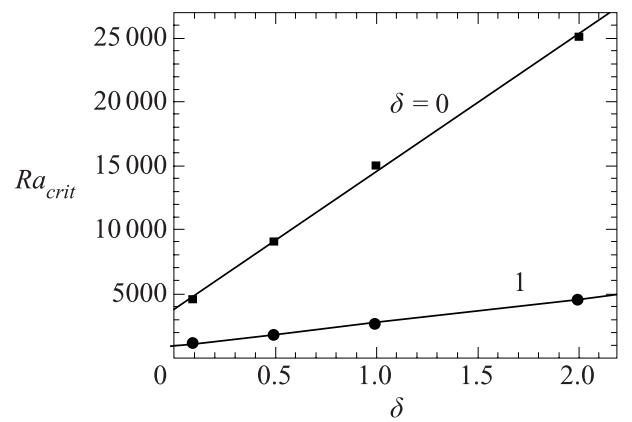

FIGURE 17. The critical Rayleigh number $R a_{\text {crit }}$ as a function of the interfacial thickness parameter $\delta$, for the axisymmetric mode $\beta=0$ and the most dangerous three-dimensional mode $\beta=1$. For all values of $\delta$, the axisymmetric mode is seen to have a larger value of $R a_{c r i t}$.

critical Rayleigh number $R a_{c r i t}$ as a function of $\delta$, for $\beta=1$ and $\beta=0$. Similarly to the axisymmetric case discussed above, by linear regression through the data points we obtain the relationship for $\beta=1$

$$
R a_{\text {crit }}=1800 \delta+920 .
$$

For all interfacial thicknesses, the value of $R a_{\text {crit }}$ for $\beta=1$ is thus lower than the corresponding value for $\beta=0$. The above relationship furthermore indicates that Rayleigh numbers below $O(920)$ are stable for all base concentration profiles, with respect to any axisymmetric or three-dimensional perturbation. By extrapolating the growth rate for $\beta=1$ and $R a=10^{7}$ to $\delta=0$, we find that the maximum eigenvalue for a step-like concentration base state is approximately 0.028 , as shown in figure 18 . For a Hele-Shaw cell, Graf et al. (2002) reported $R a_{\text {crit }}=50$ and the maximum eigenvalue to be 0.038 . This variation can be attributed to the stabilizing influence by the walls which is higher, for corresponding parameters, in a capillary tube than in a Hele-Shaw cell.

It is interesting to compare the above relationship (3.2) for the present, errorfunction-type base concentration profiles with the classical result by Taylor (1954) regarding unstable density stratifications with a constant gradient $\mathrm{d} c / \mathrm{d} z$ in a capillary 


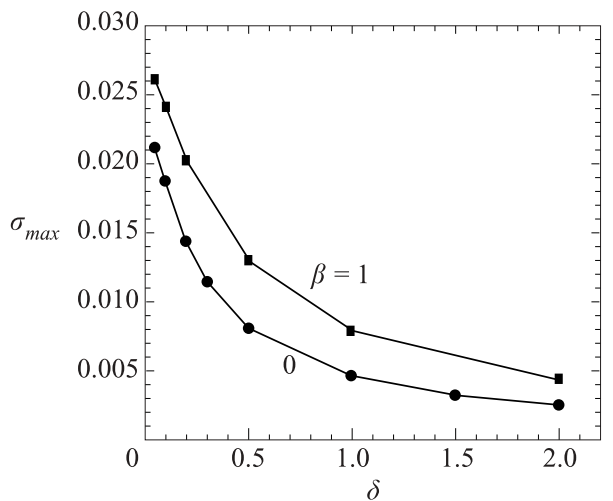

Figure 18. The growth rate $\sigma$ as a function of $\delta$, for $\beta=0$ and $\beta=1$, with $R a=10^{7}$. This indicates that for all values of the interface thickness, $\beta=1$ remains more dangerous than the axisymmetric mode.

tube. Taylor demonstrated that such profiles are stable as long as

$$
\frac{(\mathrm{d} c / \mathrm{d} z) \rho_{0} g \alpha d^{4}}{D \mu} \leqslant 1087,
$$

where

$$
\rho=\rho_{0}(1+\alpha c) \text {. }
$$

For relatively smooth base concentration profiles of the error function type, i.e. reasonably large values of $\delta$, the present criterion (3.2) should approach the classical Taylor criterion (3.3). In order to check if this is the case, we rewrite (3.2) for dimensional $\delta$ as

$$
\left(d \frac{\mathrm{d} c}{\mathrm{~d} z}\right)^{-1} \times \frac{(\mathrm{d} c / \mathrm{d} z) \rho_{0} g \alpha d^{4}}{D \mu} \leqslant 1800 \frac{\delta}{d}+920 .
$$

In the first term on the left-hand side, we approximate $\mathrm{d} c / \mathrm{d} z$ with the value at $z=0$ :

$$
\frac{\mathrm{d} c}{\mathrm{~d} z}(z=0)=\frac{1}{\delta \sqrt{\pi}} .
$$

For $\delta / d=2$, we thus obtain

$$
\frac{(\mathrm{d} c / \mathrm{d} z) \rho_{0} g \alpha d^{4}}{D \mu} \leqslant 1275,
$$

which is indeed close to the relationship derived by Taylor, (3.3). The slightly higher value compared to Taylor's result is expected, since we based the comparison on the largest value of the concentration gradient, rather than its average value.

The variation of the growth rate $\sigma$ with $R a$, for different $\delta$ and $\beta=1$, is shown in figure 19. While this graph has the same qualitative shape as figure 4 for the axisymmetric case in that it reaches a plateau for large $R a$, the eigenvalues are consistently higher. Furthermore, for the same value of $\delta$ the unstable range extends to lower values of $R a$. The cutoff wavenumber $\beta_{\text {crit }}$ is shown in figure 20 as a function of the Rayleigh number, for various interface thickness parameters. The dashed line suggests that in the limit of large $R a$ values, $\beta_{\text {crit }}$ grows approximately in proportion to $R a^{1 / 3}$. This exponent is very close to the value of $10 / 33$ found by Graf et al. (2002) for the corresponding Hele-Shaw problem. 


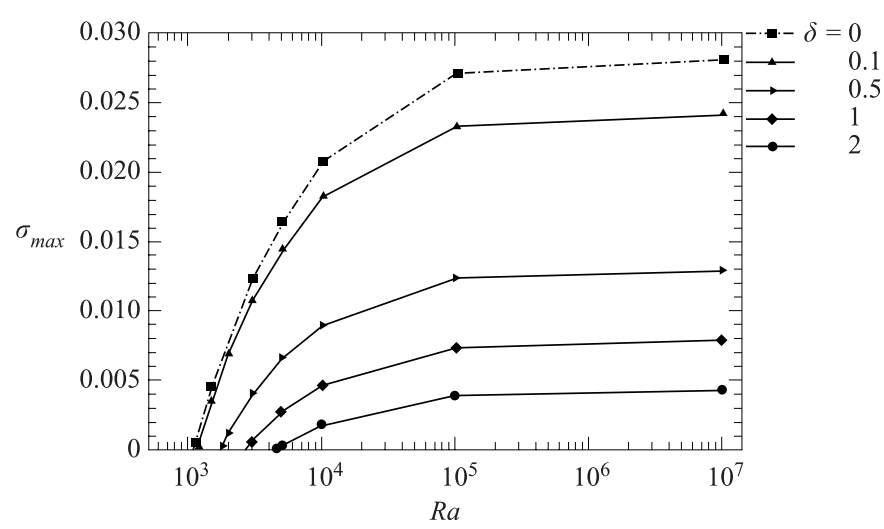

FIGURE 19. The growth rate $\sigma$ corresponding to the most dangerous wavenumber $\beta=1$, as a function of $R a$ for different interface thicknesses. The dashed line corresponds to extrapolated data for the step-function profile.

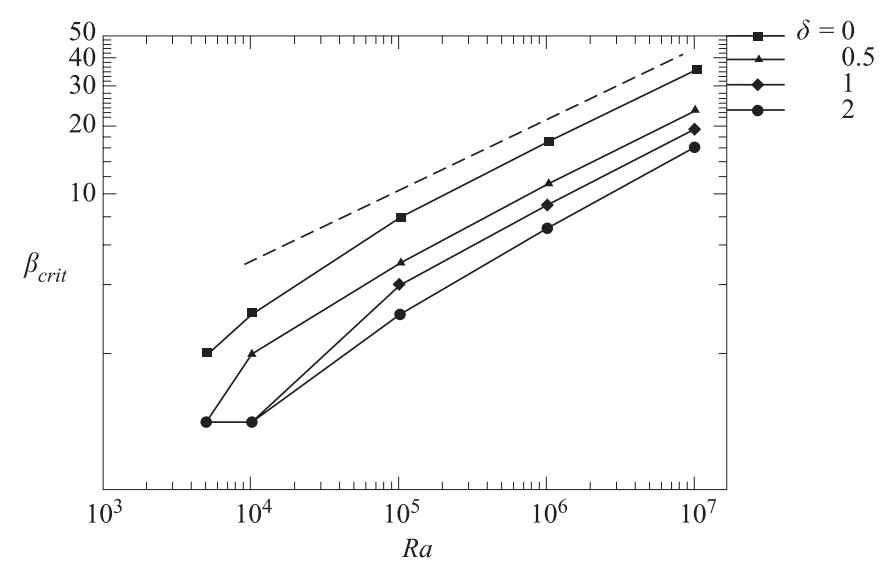

FIGURE 20. Cutoff wavenumber $\beta_{\text {crit }}$ as a function of $R a$ for various $\delta$. The dashed line represents the power law $\beta_{\text {crit }} \approx R a^{0.33}$.

\section{Influence of net axial flow}

In this section, we will set up the framework for analysing the effect of a small net axial velocity on the linear stability of the miscible interface in the configuration considered above. Our approach builds on the findings of Taylor (1953), who analysed the dispersion of a passive solute in a solvent flowing slowly through a tube. For a Poiseuille flow profile of the form

$$
\bar{q}_{z}=u_{\max }\left(1-4 r^{2} / d^{2}\right)
$$

he showed that radial flow-induced concentration variations are rendered small by the action of molecular diffusion when

$$
\frac{\delta}{u_{\max }} \gg \frac{d^{2}}{7.6^{2} D} .
$$

This can be expressed as a condition on the Péclet number:

$$
P e=\frac{u_{\max } d}{D} \ll 7.6^{2} \frac{\delta}{d} .
$$


Note that $P e$ is related to $R a$ as

$$
P e=R a \frac{u_{\max }}{U^{*}}=R a u_{n}
$$

with $U^{*}$ as defined in (2.6). Under these circumstances, Taylor (1953) shows that the dimensionless concentration profile of the passive scalar evolves according to

$$
c(r, \xi)=0.5+0.5 \operatorname{erf}\left(\frac{\xi}{2 \sqrt{k t}}\right)+\frac{P e}{16 \sqrt{\pi k t}}\left(r^{2}-2 r^{4}\right) \exp \left(-\frac{\xi^{2}}{4 k t}\right) .
$$

Here $\xi=z-\frac{1}{2} u_{\max } t$ denotes the axial coordinate in a reference frame moving with the mean velocity $\frac{1}{2} u_{\max }$ of the flow; $k=u_{\max }^{2} d / 768 D$ represents the well-known Taylor dispersion coefficient.

In order to investigate the influence of this Poiseuille flow on the stability of the interface, we can now set

$$
\delta=2 \sqrt{k t}
$$

and assume a quasi-steady state $\delta=$ const., in complete correspondence to the no-flow case analysed above. However, when interpreting the results it needs to be kept in mind that the situation under consideration, in which the density depends on the concentration, is clearly distinct from the case of a passive scalar analysed by Taylor (1953). As a result, the flow is not exactly of Poiseuille type. Nevertheless, as long as radial concentration variations remain small, the Poiseuille flow assumption should be approximately valid.

The set of linear equations (2.24)-(2.28) now has to be modified in order to account for the axial base flow and the radial variations in the concentration profile. After linearization, these effects appear only in the concentration equation, which in the moving reference frame changes to

$$
\sigma \hat{c}+\frac{1}{r} \frac{\partial \hat{\psi}}{\partial r} \frac{\partial \bar{c}}{\partial \xi}+\bar{q}_{z} \frac{\partial \hat{c}}{\partial \xi}-\frac{1}{r} \frac{\partial \hat{\psi}}{\partial \xi} \frac{\partial \bar{c}}{\partial r}=\frac{1}{R a}\left(\frac{\partial^{2} \hat{c}}{\partial r^{2}}+\frac{1}{r} \frac{\partial \hat{c}}{\partial r}+\frac{\partial^{2} \hat{c}}{\partial \xi^{2}}\right)
$$

for axisymmetric perturbations, and to

$$
\sigma \hat{c}+\hat{q}_{z} \frac{\partial \bar{c}}{\partial \xi}+\bar{q}_{z} \frac{\partial \hat{c}}{\partial \xi}+\frac{\hat{q}_{r}}{r} \frac{\partial \bar{c}}{\partial r}=\frac{1}{R a}\left(\frac{1}{r} \frac{\partial}{\partial r}\left(r \frac{\partial \hat{c}}{\partial r}\right)-\frac{\beta^{2}}{r^{2}} \hat{c}+\frac{\partial^{2} \hat{c}}{\partial \xi^{2}}\right)
$$

for the three-dimensional case. In the following, numerical stability results will be presented for the dimensionless parameters $R a=3 \times 10^{4}$ and $\delta=2$, at various values of $u_{n} \leqslant 0.0005$. In this way, $P e \leqslant 15$, so that condition (4.3) is clearly satisfied. Figure 21 shows the corresponding base concentration profile. Clearly, radial concentration variations are small.

Figure 22 displays the variation of the growth rate with the normalized velocity $u_{n}$ for both the axisymmetric and the $\beta=1$ azimuthal mode. The growth rates of both modes show a small decrease with increasing net flow. However, for the present low flow rates there is no indication that the growth rate of the axisymmetric mode will soon overtake that of the $\beta=1$ mode. For reasons that are not completely understood, we were unable to obtain fully converged eigenfunctions for flow rates much larger than those shown in figure 22. The corresponding eigenfunctions for the $\beta=1$ and 0 modes are shown in figures 23 and 24. For $R a=3 \times 10^{4}$ and $\delta=2$, eigenfunctions with no axial flow are very similar to figures 7 and 12 for the axisymmetric and azimuthal perturbations. Comparing the corresponding figures clearly shows the convective effect of the base flow on the concentration perturbation field. 


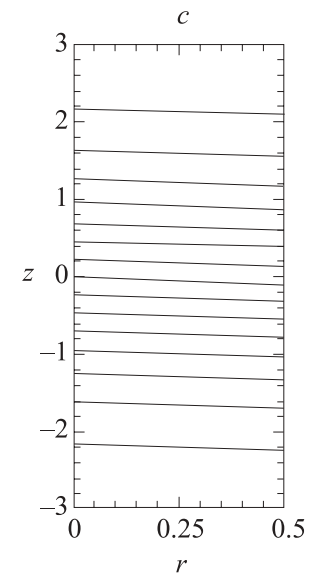

FIGURE 21. Influence of net axial flow on the interfacial stability. Base concentration profile $\bar{c}$ for $R a=3 \times 10^{4}, \delta=2$ and $u_{n}=0.0002$.

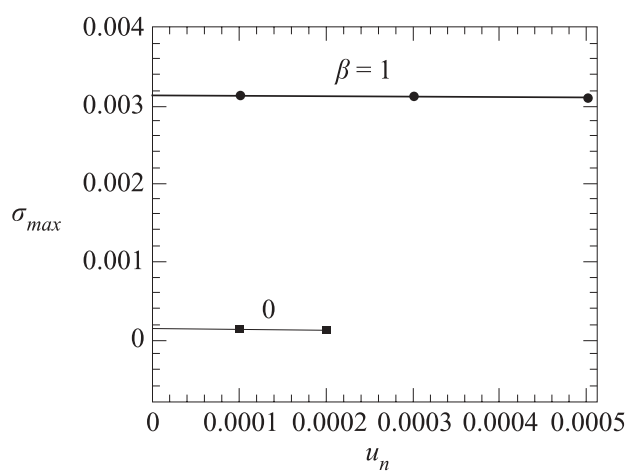

FIGURE 22. Influence of net axial flow on the interfacial stability. Variation of instability growth rates for $R a=3 \times 10^{4}, \delta=2$ and various values of $u_{n}$, for axisymmetric perturbations $\beta=0$, as well as for three-dimensional perturbations with $\beta=1$. The axial flow is seen to result in a small reduction of the growth rates.
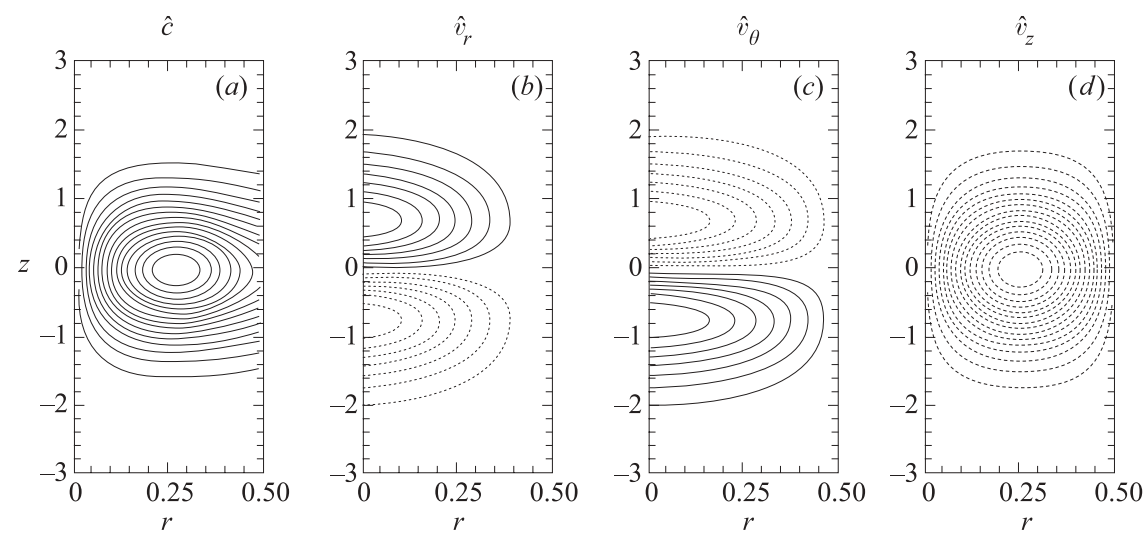

FIGURE 23. Influence of net axial flow on the interfacial stability. Concentration and velocity eigenfunction contours for $\beta=1$, at $R a=3 \times 10^{4}, \delta=2$ and $u_{n}=0.0002$. 

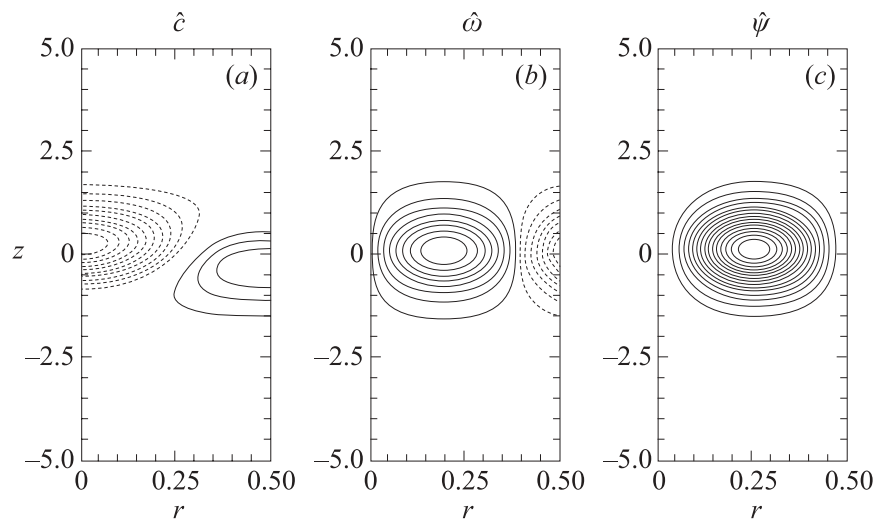

FIGURE 24. Influence of net axial flow on the interfacial stability. Concentration, vorticity and streamfunction eigenfunction contours for $\beta=0$, at $R a=3 \times 10^{4}, \delta=2$ and $u_{n}=0.0002$.

\section{Conclusions}

The current investigation presents linear stability results for the miscible interface formed by placing a heavier fluid above a lighter one in a vertically oriented capillary tube. The analysis is based on the three-dimensional Stokes equations coupled to a convection-diffusion equation for the concentration field in cylindrical coordinates. By linearizing this set of equations, a generalized eigenvalue problem is formulated, whose numerical solution yields both the growth rate and the two-dimensional eigenmodes as functions of the dimensionless parameters characterizing the problem, namely the Rayleigh number and the interface thickness.

The dispersion relations show that for all $R a$ values and interface thicknesses the azimuthal mode $\beta=1$ represents the most unstable disturbance. In particular, its growth rates are consistently higher than those of the axisymmetric mode. The most amplified mode thus corresponds to the formation of one finger of the lighter fluid rising over one half of the tube's cross-section, with a second finger of the heavier fluid falling in the other half. This is in agreement with the experimental observations of Kuang et al. (2002). The stability results furthermore indicate the existence of a critical Rayleigh number $R a_{\text {crit }} \approx 920$, below which all perturbations are stable. For relatively thick interfaces, the present data for $R a_{c r i t}$ are seen to approach the classical value derived by Taylor (1954) for a uniform density gradient. For a constant interface thickness, the growth rates reach a plateau as $R a>10^{6}$.

The experiments of Kuang et al. (2002) had shown that a small amount of net flow through the capillary tube can stabilize the azimuthal instability mode and maintain an axisymmetric evolution of the flow. In order to analyse the effect of a net flow on the linear stability of the interface, we included a base flow of Poiseuille type in our analysis. The corresponding base concentration profile was obtained following the analysis of the convection and diffusion of a passive scalar in a capillary tube by Taylor (1953). The stability analysis shows that a weak base flow results in a slight reduction of the growth rates of both axisymmetric and azimuthal modes. However, within the velocity interval for which we were able to obtain converged results there is no indication that the axisymmetric mode will overtake its azimuthal counterpart. Consequently, we are at present unable to state conclusively if the experimental observations by Kuang et al. (2002) reflect the influence of the base flow on the linear stability problem, or if they represent a nonlinear effect. In addition, it is to be kept in mind that the assumption of a Poiseuille-type base flow is only an 
approximation, which requires a relatively thick interface. While it is unknown what the initial interface thickness in the experiments was, we do know that the experiments employed fluids of very different viscosities, an effect that has not been accounted for in the present analysis. Clearly, further work is needed in order to unravel these issues.

We thank Professors Tony Maxworthy and Bud Homsy for several helpful discussions. Support for this research was received from the NASA Microgravity and NSF/ITR programs, as well as from the Department of Energy, and through an NSF equipment grant.

\section{REFERENCES}

Batchelor, G. K. \& NitsChe, J. M. 1991 Instability of stationary unbounded stratified fluid. J. Fluid Mech 227, 357.

BAtCheloR, G. K. \& Nitsche, J. M. 1993 Instability of stratified fluid in a vertical cylinder. J. Fluid Mech 252, 419.

Chen, C.-Y. \& Meiburg, E. 1996 Miscible displacements in capillary tubes. Part 2. Numerical simulations. J. Fluid Mech. 326, 57.

Chen, C.-Y. \& Meiburg, E. 2002 Miscible displacements in capillary tubes. Influence of Korteweg stresses and divergence effects. Phys. Fluids 14, 2052.

Cox, B. G. 1962 On driving a viscous fluid out of a tube. J. Fluid Mech. 14, 81.

Debacq, M., Fanguet, V., Hulin, J. P. \& Salin, D. 2001 Self-similar concentration profiles in buoyant mixing of miscible fluids in a vertical tube. Phys. Fluids 13, 3097.

Fernandez, J., Kurowski, P., Petitjeans, P. \& Meiburg, E. 2002 Density-driven, unstable flows of miscible fluids in a Hele-Shaw cell. J. Fluid Mech. 451, 239.

Fletcher, C. A. J. 1991 Computational Techniques for Fluid Dynamics. Springer.

Graf, F., Meiburg, E. \& Härtel, C. 2002 Density-driven instabilities of miscible fluids in a HeleShaw cell: Linear stability analysis of the three-dimensional Stokes equations. J. Fluid Mech. 451, 261.

Hales, A. L. 1937 Convection currents in geysers. Mon. Not. R. Astron. Soc., Geophys. Suppl. 4, 122.

Joseph, D. \& Renardy, Y. 1992 Fundamentals of Two-Fluid Dynamics. Springer.

KorTeWEG, D. 1901 Sur la forme que prennent les équations du mouvement des fluides si l'on tient compte des forces capillaires causées par des variations de densité. Arch. Neel. Sci. Ex. Nat. II, 6 .

Kuang, J., Maxworthy, T. \& Petitjeans, P. 2002 Miscible displacements in cylindrical tubes: velocity fields and streamline patterns. Eur. J. Mech. B 22, 271.

Maschhoff, M. J. \& Sorensen, D. C. 1996 P-ARPACK: An efficient portable large scale eigenvalue package for distributed memory parallel architectures. In Applied Parallel Computing in Industrial Problems and Optimization. Lecture Notes in Computer Science, vol. 1184. Springer.

Petitjeans, P. \& Maxworthy, T. 1996 Miscible displacements in capillary tubes. Part 1. Experiments. J. Fluid Mech. 326, 37.

Reinelt, D. A. \& Saffman, P. G. 1985 The penetration of a finger into a viscous fluid in a channel and tube. SIAM J. Sci. Statist. Comput. 6, 542.

SCOFfoni, J., Lajeunesse, E. \& Homsy, G. M. 2000 Interface instabilities during displacements of two miscible fluids in a vertical pipe. Phys. Fluids 13, 553.

Soares, E. J., Carvalho, M. S. \& Souza Mendes, P. R. 2002 Immiscible liquid-liquid displacement in capillary tubes. Preprint.

Sorensen, D. C. 1992 Implicit application of polynomial filters in a k-step Arnoldi method. SIAM J. Matrix Anal. Appl. 13, 357.

TAYLOR, G. I. 1953 Dispersion of soluble matter in solvent flowing slowly through a tube. Proc. R. Soc. Lond. A 219, 186.

TAYLOR, G. I. 1954 Diffusion and mass transport in tubes. Proc. Phys. Soc. B 67, 857.

TAYLOR, G. I. 1960 Deposition of a viscous fluid on the wall of a tube. J. Fluid Mech. 10, 161. 
Verzicco, R. \& Orlandi, P. A. 1996 A finite-difference scheme for three-dimensional incompressible flows in cylinder coordinates. J. Comput. Phys 123, 402.

Wilhelm, D. \& Meiburg, E. 2003 Three-dimensional spectral element simulations of variable density and viscosity, miscible displacements in a capillary tube. Computers and Fluids (to appear).

Wooding, R. A. 1959 The stability of a viscous liquid in a vertical tube containing porous material. Proc. R. Soc. Lond. A 252, 120. 